\title{
(2) OPEN ACCESS \\ Candida glabrata chorioamnionitis in a twin pregnancy following in vitro fertilisation: a rare cause of septic miscarriage
}

\author{
Muhammad Ashraf Yusoff $\odot,{ }^{1}$ lan Liang En Wee, ${ }^{2}$ Jean Xiang Ying Sim, ${ }^{2}$ Liying Yang ${ }^{1}$
} Singapore General Hospital, Singapore

2Infectious Diseases, Singapore General Hospital, Singapore

\section{Correspondence to} Dr Muhammad Ashraf Yusoff; mdashrafyusoff@gmail.com

Accepted 30 December 2021
'Obstetrics and Gynaecology,

\section{SUMMARY}

Candida glabrata chorioamnionitis is uncommon and associated with poor fetal outcomes. We present a case of $C$. glabrata chorioamnionitis of a dichorionic diamniotic pregnancy conceived by fresh embryo transfer. Despite antifungal therapy with amphotericin $B$, spontaneous miscarriage of both twins occurred. The mother otherwise recovered uneventfully. Our case highlights the high fetal mortality associated with C. glabrata chorioamnionitis and the challenges of antifungal treatment in pregnancy. We recommend a low threshold of suspicion for Candida infection in pregnancy, notably those involving transvaginal uterine instrumentation such as in vitro fertilisation.

\section{BACKGROUND}

Candida glabrata chorioamnionitis is reported to be associated with uterine instrumentation and assisted reproductive techniques, ${ }^{1}$ and may result in poor fetal outcomes. This report describes our experience with a case of culture and histology-proven C. glabrata chorioamnionitis in a dichorionicdiamniotic twin pregnancy conceived via in vitro fertilisation (IVF). We highlight our clinical dilemmas in the management of this case, particularly in counselling of the risk of continuing the pregnancy and selection of the appropriate antifungal agents and duration of therapy.

\section{CASE PRESENTATION}

Our patient was gravida 1 with dichorionic, diamniotic twins conceived via fresh embryo transfer (FET). An endometrial scratch performed prior to the FET showed no endometritis.

She presented with premature rupture of membranes (PROM) at $17+4$ weeks' gestation (Day 0). She was febrile at $38.5^{\circ} \mathrm{C}$ and inflammatory markers were raised with a $\mathrm{C}$ reactive protein

Check for updates

(c) BMJ Publishing Group Limited 2022. Re-use permitted under CC BY-NC. No commercial re-use. See rights and permissions. Published by BMJ.

To cite: Yusoff MA, Wee ILE, Sim JXY, et al. BMJ Case Rep 2022;15:e246629. doi:10.1136/bcr-2021246629

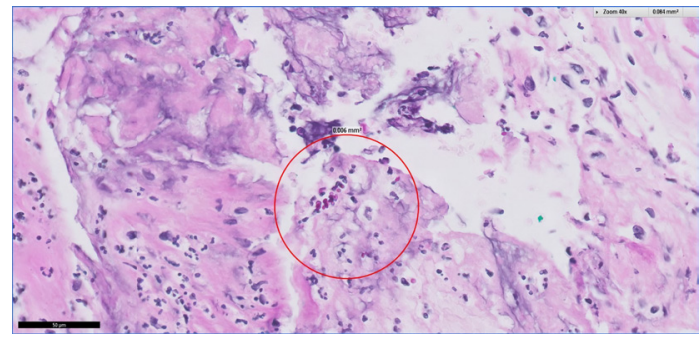

Figure 1 Histology of placental membranes, showing necrotising chorioamnionitis (fungi highlighted).

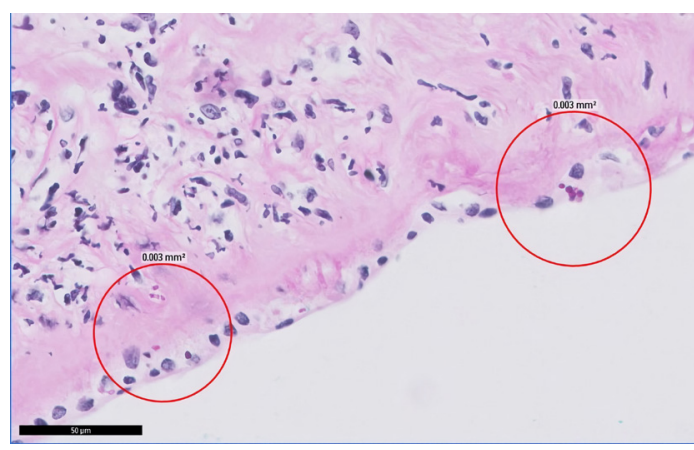

Figure 2 Histology of umbilical cord, showing funisitis (fungi highlighted).

(CRP) of $57.7 \mathrm{mg} / \mathrm{L}(0.2-9.1 \mathrm{mg} / \mathrm{L})$ and white blood cell count (TW) of $17.5 \times 10^{9} / \mathrm{L}\left(4-10 \times 10^{9} / \mathrm{L}\right)$. Ultrasound showed two viable fetuses and oligohydramnios (AFI 2.5) of the presenting twin. Empiric intravenous antibiotics (ceftriaxone and metronidazole) were initiated.

\section{INVESTIGATIONS}

Maternal blood cultures and high vaginal swabs obtained on admission grew C. glabrata on Day 2. The patient was counselled by both the maternalfetal medicine and infectious diseases teams regarding the suspicion of fungal chorioamnionitis resulting in PROM, and termination of pregnancy was discussed in view of the poor fetal prognosis and risk of worsening maternal sepsis. However, she elected to continue expectant management with close monitoring. Diagnostic amniocentesis was not performed given its attendant risks. An echocardiogram to rule out cardiac seeding and a detailed ophthalmological examination to exclude endophthalmitis were both negative.

Cultures of both placentas grew C. glabrata, while histological examination showed acute chorioamnionitis (figure 1) and funisitis (figure 2), with fungal yeasts seen at the membranes and amnion of the umbilical cord. Sensitivity testing of C. glabrata showed sensitivity to anidulafungin (minimum inhibitory concentration (MIC) $0.12 \mu \mathrm{g} /$ $\mathrm{mL}$ ) and caspofungin (MIC $0.12 \mu \mathrm{g} / \mathrm{mL}$ ), and dosedependent susceptibility to fluconazole (MIC 32 $\mu \mathrm{g} / \mathrm{mL})$. No interpretive criteria were available for sensitivity to amphotericin B.

\section{TREATMENT}

Given maternal fungemia, fungal chorioamnionitis was suspected and intravenous amphotericin B was 


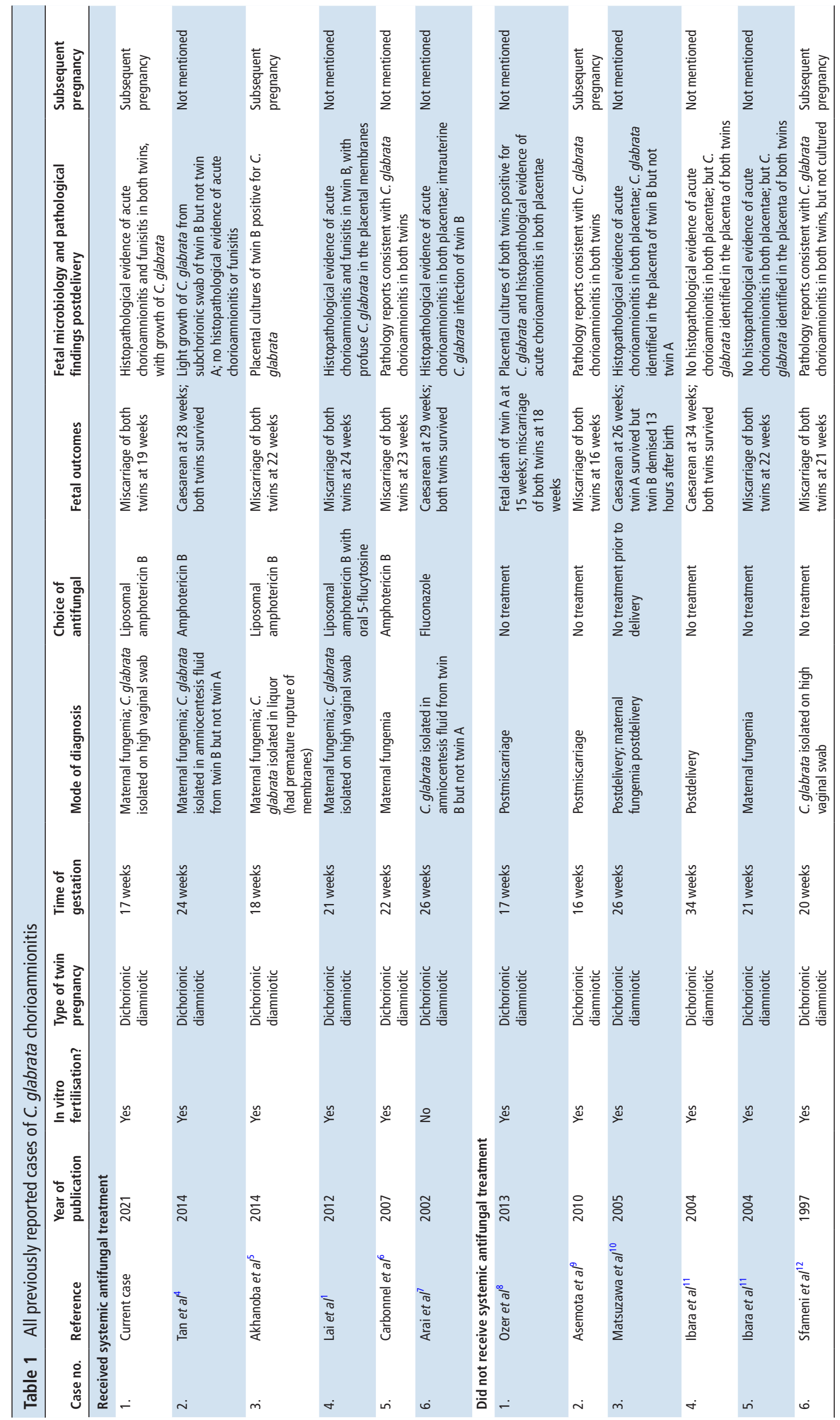


started on Day 2. The patient's temperature decreased and she improved both clinically and biochemically with a response in her CRP levels. At Day 11, despite 9 days of appropriate antifungal therapy, the patient developed vaginal spotting. Speculum examination revealed a fully dilated cervical os and fetal head at station -1 in the vagina. This progressed to a spontaneous miscarriage of the leading twin. The couple requested to continue expectant management for the second twin; the cord of the first twin was thus tied off and the placenta was left in situ. At Day 13, recrudescence of fever occurred, accompanied by miscarriage of the second twin and both placentas.

\section{OUTCOME AND FOLLOW-UP}

Postdelivery, intravenous amphotericin B was switched to intravenous anidulafungin to reduce the risk of nephrotoxicity. Our patient improved clinically and her TW and CRP counts rapidly normalised. She completed a 2-week course of intravenous antifungals from her first negative blood culture.

\section{DISCUSSION}

C. glabrata is generally considered to be an innocuous vaginal commensal and rarely associated with pathogenicity in healthy, non-immunocompromised individuals. ${ }^{2}$ This reduced virulence is mainly due to the inability of C. glabrata to produce hyphae and its less adhesive capacity, thereby reducing its capability to invade across intact chorionic membranes. ${ }^{3}$ C. glabrata chorioamnionitis is a rare entity; in a 2015 review, only 20 cases were identified in the literature. ${ }^{3}$ There is, however, a strong link between C. glabrata chorioamnionitis and IVF and invasive uterine instrumentation, ${ }^{2}$ supporting a possible iatrogenic mechanism of pathogenesis, in which C. glabrata chorioamnionitis and subsequently sepsis are facilitated by direct uterine inoculation via antenatal instrumentation. Given the association with IVF, most reported cases of C. glabrata chorioamnionitis have involved twin pregnancies. ${ }^{23}$ To identify all previously reported cases in the medical literature of C. glabrata chorioamnionitis in twin pregnancies, we searched (without language restrictions) the following computerised databases using the terms 'Candida', 'Torulopsis', 'glabrata', 'chorioamnionitis', 'congenital', 'perinatal' and 'infection': MEDLINE and PubMed from January 1980 to December 2021. After excluding all cases involving singleton pregnancies, our search strategy identified 10 papers included by abstract evaluation. Of the 10 papers identified, some included two case reports, and the number of cases eventually analysed was 12 (including the case reported in our institution). Cases were reported between 1997 and 2021 (table 1). ${ }^{4-12}$

Notably, almost all cases occurred in the second trimester, suggesting a possible latent incubation phase between antenatal instrumentation and presentation of frank infection. ${ }^{4}$ Our case presented at $17+4$ weeks, which posed a therapeutic dilemma in management at the point of presentation-to advocate for termination of pregnancy given the significant fetal mortality for presumed fungal chorioamnionitis, or to continue treating the presumptive chorioamnionitis by administering systemic maternal antifungal treatment with their attendant potential drug toxicities, till fetal viability could be attained. ${ }^{45}$

In the case of C. glabrata chorioamnionitis, administering systemic maternal antifungal treatment is a non-trivial undertaking, as the optimal choice and duration of antenatal antifungal therapy for this unusual organism is yet to be clearly defined. Fetal considerations also further complicate antimicrobial choice. As C. glabrata commonly exhibits inherent resistance to azoles such as fluconazole, higher doses are required for effective treatment. However, high doses of azoles in pregnancy (400-600 mg/day) are contraindicated due to potential fetotoxicity (FDA Category D). ${ }^{13}$ The echinocandin class of antifungals which include anidulafungin have limited human data regarding safety in pregnancy, with teratogenic effects reported in animal studies (FDA Category C). ${ }^{13}$ Polyenes such as amphotericin B are generally considered the safest for the treatment of C. glabrata sepsis in pregnancy (Category $\mathrm{B})^{13}$; the placenta tissue also appears to act as a reservoir for slow release of amphotericin $\mathrm{B}$ into the intrauterine environment. ${ }^{14}$ However, use of amphotericin B is associated with significant side effects such as nephrotoxicity and electrolyte imbalances. ${ }^{15}$ In our review of the literature, $50.0 \%(6 / 12)$ of the cases received systemic maternal antifungal treatment, ${ }^{14-7}$ with the majority treated with amphotericin B. ${ }^{4-6}$ Maternal candidaemia, such as occurred in our case, was an additional indication for systemic antifungal treatment. The majority of cases with maternal candidaemia were treated with amphotericin $\mathrm{B}^{14-6}$ as well. In the two cases of untreated maternal candidaemia, candidaemia occurred shortly before or just after delivery. ${ }^{10} 11$ No cases of maternal mortality were reported.

Unfortunately, C. glabrata chorioamnionitis remains associated with poor fetal outcomes and substantial fetal mortality. In the literature, only one-third (4/12) of cases had successful outcomes, with equal numbers in both treated and untreated groups. ${ }^{4} 1011$ In both cases that reported fetal survival posttreatment with systemic maternal antifungal treatment, caesarean delivery was performed soon after diagnosis at $28^{4}$ and 29 weeks. ${ }^{7}$ One case was successfully treated with amphotericin $\mathrm{B}^{4}$ and the other with fluconazole. ${ }^{7}$ Furthermore, in both cases, C. glabrata was only isolated in amniocentesis fluid from a solitary fetus but not the other, ${ }^{4}$ suggesting a potential association between survival and lower burden of disease. Unfortunately, in our case, amniocentesis could not be performed for prognostication due to presentation with PROM resulting in oligohydramnios of the presenting twin. The extensive burden of fungal infection was only confirmed on histopathological examination after miscarriage.

Notably, cases with fetal survival generally presented at a more advanced stage of pregnancy (24-34 weeks). ${ }^{410} 11$ In our case, despite continuation of systemic maternal antifungals for $\geq 1$ week and clearance of fungemia in maternal blood cultures, septic miscarriage of both twins still occurred. This may be attributed to presentation at an earlier phase of gestation, as well as extensive burden of fungal infection. Histopathological examination revealed evidence of acute chorioamnionitis and funisitis in both twins, with growth of C. glabrata. In the literature, either histopathological evidence of chorioamnionitis or growth of C. glabrata was reported in both twins in a majority $(8 / 12)$ of cases. ${ }^{6-12}$ Our case was one of only two in the literature that reported both histopathological evidence of chorioamnionitis as well as retrieval of the causative organism (C. glabrata) from both twins. ${ }^{8}$

Screening and treatment of Candida infection prior to embryo transfer and during antenatal visits may be considered for IVF pregnancies, ${ }^{9}$ given the association with C. glabrata infections and the high financial and emotional cost of losing an IVF pregnancy. This should be undertaken on an individualised basis considering patient factors, extent of infection as well as risks associated with antifungal therapy. Women with C. glabrata chorioamnionitis should be managed by a multidisciplinary team to determine the optimal dose regime of antifungals and advise on timing of delivery. Even with early recognition and institution of appropriate antifungals, our case highlights the high fetal 
mortality associated with this uncommon infection, especially during the previable period. In our case, despite institution of treatment, fetal mortality was likely inevitable given presentation in an earlier phase of gestation, as well as an extensive burden of fungal infection.

\section{Learning points}

- In pregnancies involving in vitro fertilisation (IVF) or invasive antenatal procedures, a low threshold for suspicion of Candida infection should be considered. Routine screening for Candida infection may be considered for IVF pregnancies, given the high emotional and financial cost of losing an IVF pregnancy.

- Candida glabrata infections in pregnancy pose a unique challenge to treatment, as higher azole doses are typically required for effective treatment due to inherent resistance, yet high doses of azoles are also contraindicated in pregnancy due to potential fetotoxicity. Amphotericin B has the best safety profile among antifungals, but its efficacy for improving fetal outcomes is not proven.

- A multidisciplinary approach is crucial in the management of $C$. glabrata infections in pregnancy to determine the optimal dose and duration of antifungals, as well as the timing of delivery. Careful counselling and monitoring are particularly important in cases of systemic Candidasis, where prolongation of the pregnancy should not come at the expense of worsening maternal sepsis.

- C. glabrata chorioamnionitis is associated with poor fetal outcomes and substantial fetal mortality, particularly with infection at early gestation. In this case of $C$. glabrata chorioamnionitis in a twin pregnancy, fetal mortality was likely inevitable despite treatment, given presentation earlier in gestation as well as extensive fungal infection that was only diagnosed on histopathological examination subsequent to miscarriage.

Acknowledgements The authors would like to thank Dr Yu Su Ling, Senior Consultant, Department of Obstetrics and Gynaecology, Singapore General Hospital, for her expert advice in the clinical care of the patient and preparation of the report. The authors would like to thank Dr Ronald Goh Chin Hong, Department of Pathology, Singapore General Hospital, for providing the histological images in this case report.

Contributors Performed data acquisition and writing of the report: MAY. Made contributions to conception of the study, editing and review of the report: ILEW, JXYS, YL.
Funding The authors have not declared a specific grant for this research from any funding agency in the public, commercial or not-for-profit sectors.

Competing interests None declared.

Patient consent for publication Consent obtained directly from patient(s). Provenance and peer review Not commissioned; externally peer reviewed.

Open access This is an open access article distributed in accordance with the Creative Commons Attribution Non Commercial (CC BY-NC 4.0) license, which permits others to distribute, remix, adapt, build upon this work non-commercially, and license their derivative works on different terms, provided the original work is properly cited and the use is non-commercial. See: http://creativecommons.org/ licenses/by-nc/4.0/.

Case reports provide a valuable learning resource for the scientific community and can indicate areas of interest for future research. They should not be used in isolation to guide treatment choices or public health policy.

\section{ORCID iD}

Muhammad Ashraf Yusoff http://orcid.org/0000-0002-5022-3005

\section{REFERENCES}

1 Jackel D, Lai K. Candida glabrata sepsis associated with chorioamnionitis in an in vitro fertilization pregnancy: case report and review. Clin Infect Dis 2013;56:555-8.

2 Garcia-Flores J, Cruceyra M, Cañamares M, et al. Candida chorioamnionitis: report of two cases and review of literature. J Obstet Gynaecol 2016;36:843-4.

3 Ganer Herman H, Mevorach Zussman N, Krajden Haratz K, et al. Candida glabrata chorioamnionitis following in vitro fertilization: review of the literature. Gynecol Obstet Invest 2015;80:145-7.

4 Tan SQ, Ng OT, Khong CC. Candida glabrata sepsis associated with chorioamnionitis in an IVF twin pregnancy: should we deliver? J Obstet Gynaecol Res 2015;41:962-6.

5 Akhanoba F, MacDougall J, Mathur R, et al. Severe systemic candidiasis following immunomodulation therapy in in vitro fertilisation-embryo transfer (IVF-ET). BMJ Case Rep 2014;2014:bcr2013203202.

6 Carbonnel M, Kayem G, Luquet-Besson I, et al. [Candida glabrata chorioamnionitis following in vitro fertilization]. J Gynecol Obstet Biol Reprod 2007;36:705-8.

7 Arai $\mathrm{H}$, Goto R, Matsuda T, et al. Case of congenital infection with Candida glabrata in one infant in a set of twins. Pediatr Int 2002;44:449-50.

8 Özer E, Ünlü M, Erşen A, et al. Intrauterine fetal loss associated with Candida glabrata chorioamnionitis: report of two cases. Turk Patoloji Derg 2013;29:77-9.

9 Asemota OA, Nyirjesy P, Fox R, et al. Candida glabrata complicating in vitro pregnancy: successful management of subsequent pregnancy. Fertil Steril 2011;95:803.e1-e2.

10 Matsuzawa S, Ohyama M, Kawataki M, et al. Congenital Candida glabrata infection without specific nodules on the placenta and umbilical cord. Pediatr Infect Dis J 2005; 24:744-5.

11 Ibara AS, Marcorelles P, Le Martelot MT, et al. Two cases of systemic Candida glabrata infection following in vitro fertilization and embryo transfer. Eur J Clin Microbiol Infect Dis 2004;23:53-6.

12 Sfameni SF, Talbot JM, Chow SL, et al. Candida glabrata chorioamnionitis following in vitro fertilization and embryo transfer. Aust N Z J Obstet Gynaecol 1997;37:88-91.

13 Pilmis B, Jullien V, Sobel J, et al. Antifungal drugs during pregnancy: an updated review. J Antimicrob Chemother 2015;70:14-22.

14 Dean JL, Wolf JE, Ranzini AC, et al. Use of amphotericin B during pregnancy: case report and review. Clin Infect Dis 1994;18:364-8.

15 Moudgal VV, Sobel JD. Antifungal drugs in pregnancy: a review. Expert Opin Drug Saf 2003:2:475-83.

Copyright 2022 BMJ Publishing Group. All rights reserved. For permission to reuse any of this content visit

https://www.bmj.com/company/products-services/rights-and-licensing/permissions/

BMJ Case Report Fellows may re-use this article for personal use and teaching without any further permission.

Become a Fellow of BMJ Case Reports today and you can:

- Submit as many cases as you like

- Enjoy fast sympathetic peer review and rapid publication of accepted articles

- Access all the published articles

Re-use any of the published material for personal use and teaching without further permission

Customer Service

If you have any further queries about your subscription, please contact our customer services team on +44 (0) 2071111105 or via email at support@bmj.com.

Visit casereports.bmj.com for more articles like this and to become a Fellow 